\title{
Sonsinotrema calloti Dollfus, 1951 chez Hyla meridionalis en Camargue
}

\author{
par N. LEGER et C. COMBES \\ Faculté de Pharmacie, 4, avenue de l'Observatoire, F 75006 Paris \\ et Collège scientifique universitaire, F 66000 Perpignan.
}

\begin{abstract}
Résumé
Connu d'Afrique du Nord et plus récemment des Pyrénées Orientales chez Rana ridibunda, Sonsinotrema calloti a été retrouvé chez Hyla meridionalis en Carmargue, ce qui vient confirmer l'extension à l'Europe de l'aire du parasite.

Le nombre relativement élevé d'exemplaires examinés a permis en outre aux auteurs d'apporter une précision anatomique à la description de l'espèce.
\end{abstract}

\section{Summary}

First described in North Africa and found latterly in the Pyrénées Orientales in Rana ridibunda, Sonsinotrema calloti has been discovered as a parasite of Hyla meridionalis in Camargue. This confirms the presence of the species in Europe.

The relatively large number of examinated worms allows the authors to give some more details about their anatomy.

Décrit par Dollfus (1951) d'après des exemplaires récoltés chez Rana ridibunda par Balozet et Callot en 1938 en Tunisie, Sonsinotrema calloti (Digène, Lecithodendriidae) a été retrouvé par Combes et Gerbeaux chez le même hôte dans les PyrénéesOrientales (1970). Cette dernière mention du parasite concerne seulement 2 exemplaires sur un lot de 465 grenouilles. Il s'agit donc là d'un parasitisme qu'on pouvait croire 
jusqu'ici exceptionnel dans la partie septentrionale du bassin méditerranéen. Il n'a par ailleurs jamais été signalé au cours des nombreuses enquêtes réalisées en Europe par différents auteurs. Nous l'avons trouvé chez des Hyla meridionalis récoltées en Camargue.

Les captures ont été effectuées en juin 1971 dans une rizière située à proximité du Laboratoire d'Ecologie du C.N.R.S.. au Sambuc. L'endroit se caractérise par sa richesse en Hyla meridionalis et en Rana esculenta qui vivent en étroite cohabitation. Trente exemplaires mâles d'Hyla meridionalis ont été autopsiés. Cinq d'entre eux étaient parasités par Sonsinotrema calloti et nous ont livré au total 9 parasites.

Dans le lot examiné, nous avons également découvert deux autres Digènes : Pleurogenes claviger (Rudolphi, 1919) (5 fois) et Opisthioglyphe ranae (Fröhlich, 1791) (2 fois).

Ce parasitisme à fréquence relativement élevée dans une population d'Hyla meridionalis doit être considéré comme inhabituel. En effet, la Rainette est un Amphibien généralement indemne de Digènes. Notons en revanche l'absence, dans notre biotope, de Polystomum gallieni, Price, 1938, fréquent dans les mares du Languedoc-Roussillon et signalé jusqu'au Maroc.

La découverte de Sonsinotrema calloti en Camargue confirme que l'aire de ce Digène, tout comme celle de Sonsinotrema tacapense, intéresse, outre le Maghreb, une portion du rivage européen de la Méditerranée. Cette répartition à dominante nordafricaine, avec tendance à l'extension vers le nord, contraste avec celle des deux espèces du genre voisin, Pleurogenoides: $P$. medians et $P$. stromi. En effet, la première est répandue dans toute l'Europe et n'a jamais été signalée en Afrique et la seconde, bien qu'absente d'Europe septentrionale, est largement répandue sur les deux rives de la Méditerranée.

Nous résumons sur une carte l'état actuel des connaissances sur l'aire des Sonsinotrema et des Pleurogenoides dans la partie occidentale de la zone paléarctique.

Nous pensons par ailleurs que la localité prospectée en Camargue permettra, grâce à ses caractéristiques épidémiologiques privilégiées, l'étude du cycle biologique jusqu'ici inconnu de Sonsinotrema calloti.

Quant à la morphologie de nos exemplaires, elle correspond en tous points à la description originale de $S$. calloti et aux indications données par Combes et Gerbeaux (1970) pour l'un des exemplaires récoltés près de Perpignan. Nous noterons toutefois 


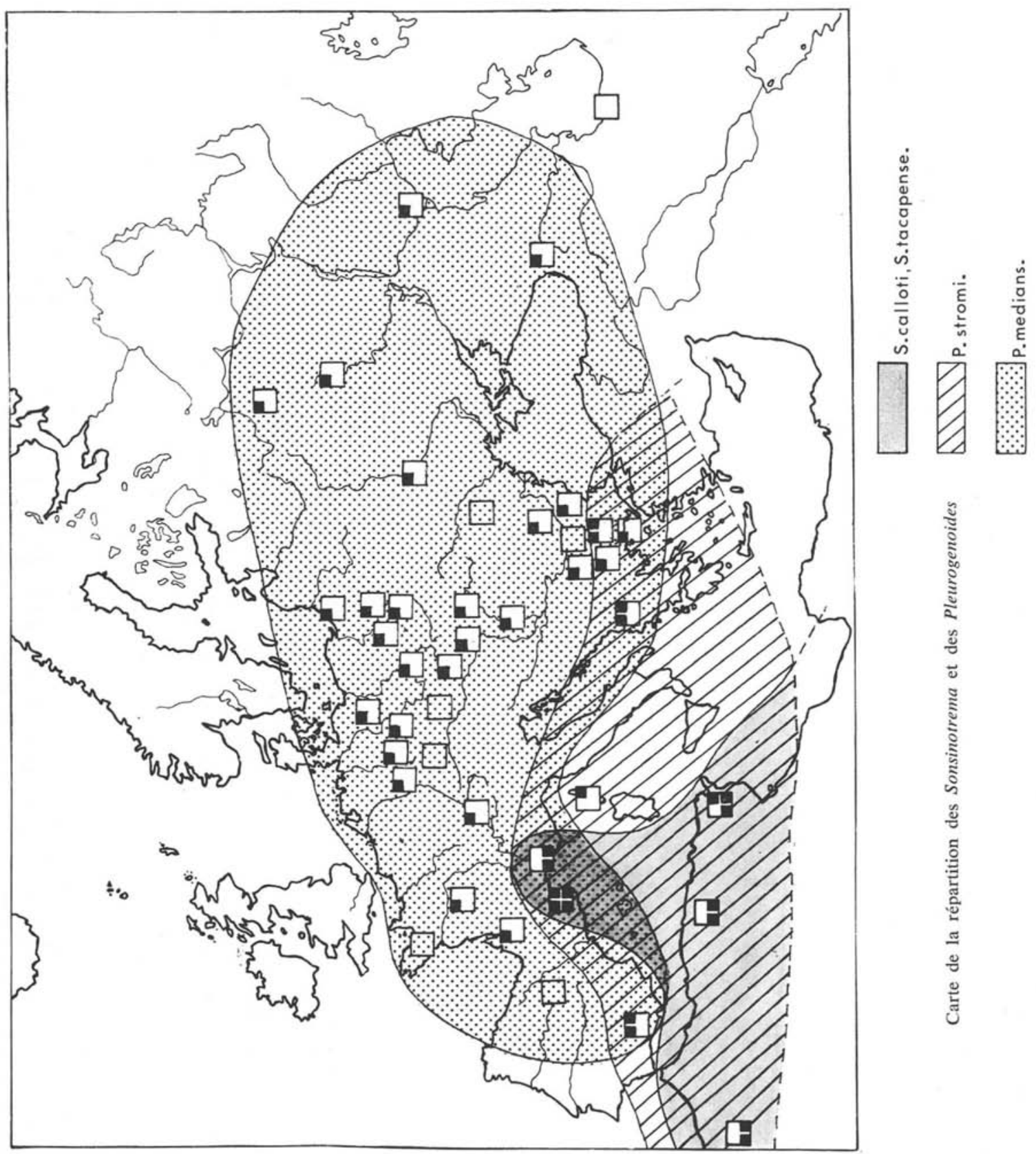




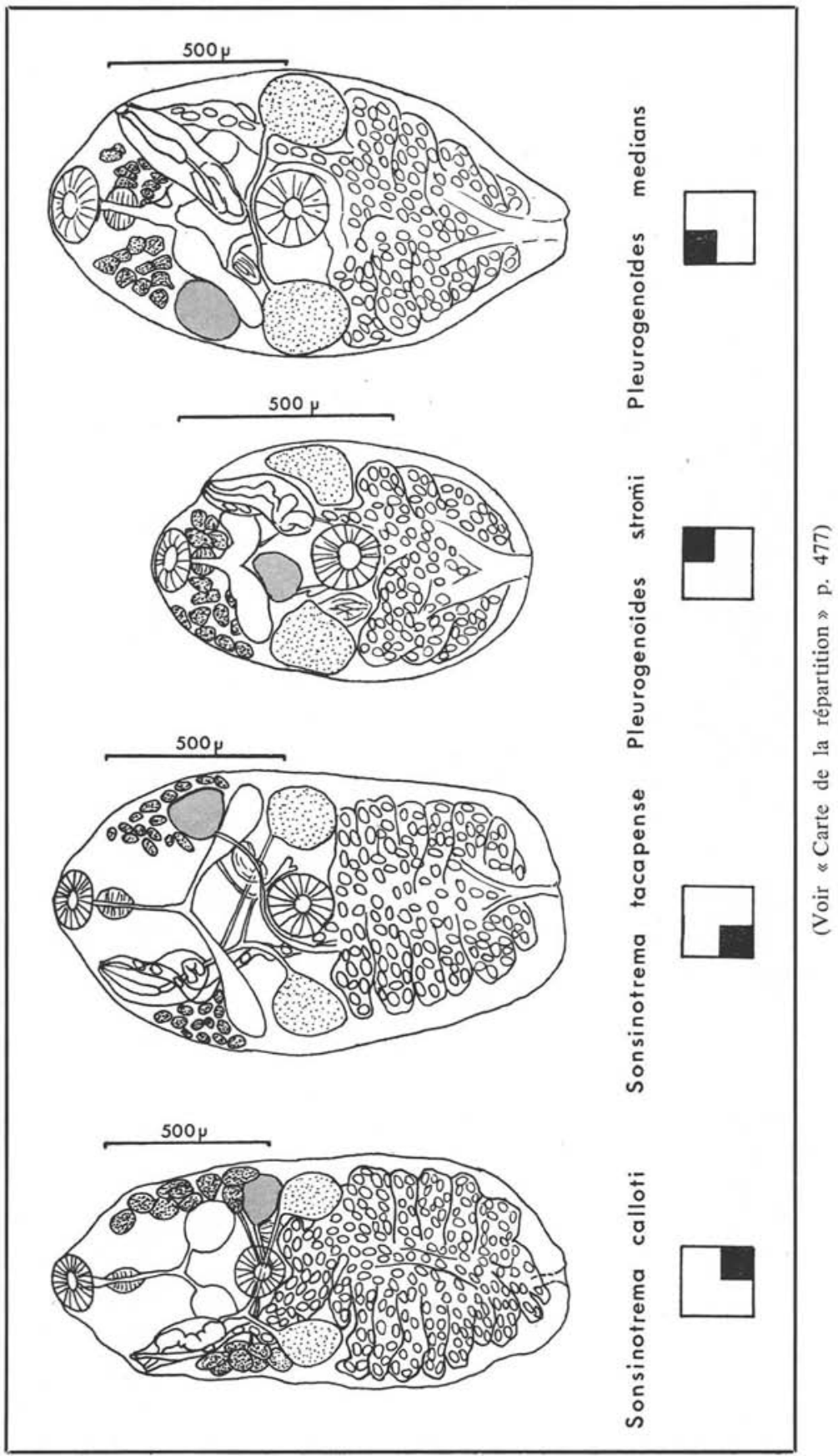


les dimensions légèrement inférieures de l'ensemble des parasites récoltés au Sambuc, comme il apparaît dans le tableau suivant:

\begin{tabular}{|c|c|c|c|}
\hline & \multirow{2}{*}{$\begin{array}{l}\text { Exemplaire récolté } \\
\text { à Perpignan }\end{array}$} & \multicolumn{2}{|c|}{ Exemplaires récoltés au Sambuc } \\
\hline & & $\mathrm{n}^{0} 1$ & $\mathrm{n}^{\circ} 2$ \\
\hline Longueur $\ldots \ldots \ldots$. & $2,20 \mathrm{~mm}$ & $1,67 \mathrm{~mm}$ & $1,51 \mathrm{~mm}$ \\
\hline Largeur $\ldots \ldots \ldots \ldots$ & $0,85 \mathrm{~mm}$ & $0,70 \mathrm{~mm}$ & $0,67 \mathrm{~mm}$ \\
\hline $\begin{array}{l}\text { Ventouse orale (dia- } \\
\text { mètre moyen) } \ldots . . .\end{array}$ & $182 \mu$ & $114 \mu$ & \\
\hline $\begin{array}{l}\text { Ventouse ventrale } \\
\text { (diamètre moyen) } \ldots\end{array}$ & $183 \mu$ & $145 \mu$ & $141 \mu$ \\
\hline Pharynx $\ldots \ldots \ldots$. & 69 sur $160 \mu$ & 79 sur $101 \mu$ & 67 sur $90 \mu$ \\
\hline Testicule côté ovaire & $274 \mu$ & $170 \mu$ & $170 \mu$ \\
\hline Testicule côté opposé & $263 \mu$ & $148 \mu$ & $170 \mu$ \\
\hline Ovaire $\ldots \ldots \ldots \ldots$. & $217 \mu$ & $159 \mu$ & $159 \mu$ \\
\hline Poche du cirre $(\mathrm{L}) \ldots$ & $389 \mu$ & $244 \mu$ & $265 \mu$ \\
\hline Eufs (longueur) $\ldots$. & 31 à $34 \mu$ & 26 à $31 \mu$ & 26 à $31 \mu$ \\
\hline
\end{tabular}

L'examen pour la première fois d'un lot assez important de ces parasites (6 exemplaires montés) nous amène à ajouter une précision, qui nous paraît importante, à la description anatomique de l'espèce. Le nombre des follicules vitellins, gros et bien individualisés, est constant : 9 côté ovaire, et 7 côté opposé (1).

Ce caractère peut être utilisé pour la diagnose différentielle avec $S$. tacapense et les espèces du genre Pleurogenoides qui possèdent toutes des follicules petits et nombreux.

\section{Bibliographie}

Balozet (L.) et Callot (J.), 1938. - Trématodes de Tunisie, I. Trématodes de Rana ridibunda (Pallas). Arch. Inst. Pasteur Tunis, 27 (1), 18-30.

Combes (C.) et Gerbeaux (M.-T.), 1970. - Recherches éco-parasitologiques sur l'helminthofaune de Rana ridibunda perezi (Amphibien anoure) dans l'est des Pyrénées. Vie et Milieu, XXI (I-C), 121-158.

Dollfus (R.-Ph.), 1951. - Miscellanea helminthologica maroccana I-III. Quelques Trématodes, Cestodes et Acanthocéphales. Arch. Inst. Pasteur Maroc, 4 (3), 104-235.

Pour une bibliographie plus complète, se reporter à l'article de Combes et Gerbeaux, 1970.

(1) Ce nombre se retrouve chez le Digène de Chauve-souris : Lecithodendrium linstowi Dollfus, 1931. 